\title{
Does Adjuvant Treatment with Chinese Herbal Medicine to Antidiabetic Agents Have Additional Benefits in Patients with Type 2 Diabetes? A System Review and Meta-Analysis of Randomized Controlled Trials
}

\author{
De Jin (D), Jiaxing Tian (D), Qi Bao, Haiyu Zhang, Qiyou Ding, \\ Fengmei Lian $(\mathbb{B}$, and Tong Xiao-lin \\ Guang An Men Hospital, China Academy of Chinese Medical Sciences, Beijing, China \\ Correspondence should be addressed to Fengmei Lian; lfm565@sohu.com and Tong Xiao-lin; tongxiaolin@vip.163.com \\ De Jin and Jiaxing Tian contributed equally to this work.
}

Received 31 October 2018; Revised 29 January 2019; Accepted 7 March 2019; Published 6 May 2019

Academic Editor: Laura De Martino

Copyright (C) 2019 De Jin et al. This is an open access article distributed under the Creative Commons Attribution License, which permits unrestricted use, distribution, and reproduction in any medium, provided the original work is properly cited.

\begin{abstract}
Introduction. In the present meta-analysis, we aimed to determine the effects of adjuvant treatment with Chinese herbal medicine (CHM) on antidiabetic agents having additional benefits in patients with type 2 diabetes. Methods. Randomized controlled trials were identified by searching the Cochrane Library, PUBMED, EMBASE, MEDLINE, the China National Knowledge Internet, Web of Science, Global Health, International Pharmaceutical Abstracts and the China biology medicine, Wanfang, and VIP databases. The intervention group received CHM as add-on treatment to antidiabetic agents therapy, and the control group received placebos in addition to antidiabetic agents or antidiabetic agents alone. We assessed pooled data, including weighted mean differences and $95 \%$ confidence intervals (CIs) using a random-effects model. Results. A total of 125 randomized controlled trials were included. 10 articles were included based on literature screening. All trials contrasted Chinese herbal medicines or Chinese herbal medicines + antidiabetic agents with placebo or antidiabetic agents + placebo and included a total of 2004 individuals with T2DM. All selected trials displayed evidence of high methodological quality and possessed a low risk of bias. Meta-analysis of the trials demonstrated that Chinese herbal medicines resulted in a more favorable blood glucose profile in contrast to placebo $(\mathrm{P}<0.05)$. The total efficacy rate differed significantly between the two groups $(\mathrm{P}<0.001)$. All ten included studies reported the occurrence of tolerable adverse effects. Conclusions. The results showed that in the intervention group, greater reductions were achieved for glucose control and body weight. The combined use of drugs improves the curative effect and has fewer adverse events and has additional benefits in patients with type 2 diabetes. This trial is registered with PROSPERO (CRD42018093867).
\end{abstract}

\section{Introduction}

Type 2 diabetes mellitus (T2DM) is a noncommunicable disease that has seen a sharp rise in its global prevalence. The latest International Diabetes Federation (IDF) survey revealed that there were approximately 425 million patients with T2DM worldwide in 2017, with this number predicted to grossly surpass the previous estimation of more than 645 million patients by 2045 . With 109.6 million patients, China is home to the largest numbers of diabetics (IDF Diabetes
Atlas.8th ed.). An epidemiological survey from China found that the estimated overall prevalence of diabetes was $10.9 \%$ amongst adults living in the country [1]. It is well known that diabetic individuals possess higher risks of comorbid illnesses, higher functional disability, and rates of premature death, compared to subjects without the condition. Patients with T2DM also suffer from a myriad of complications [2]. Several clinical trials have shown that good glycemic control is essential for maintaining the health of patients with T2DM $[3,4]$. Guidelines issued by the American Diabetes 
Association, European Association, and Chinese Diabetes Society for the Study of Diabetes recommend lifestyle interventions and oral glucose-lowering drugs (beginning with metformin) for managing HbAlc levels in patients with T2DM [5]. Additional antidiabetic medications such as sulfonylureas, thiazolidinediones, and insulin are implemented into treatment regimens when metformin monotherapy fails to achieve glycemic targets within three months [6]. Nevertheless, several of these medications possess side effects such as weight gain or hypoglycaemia that may increase insulin resistance [7].

Chinese herbal medicines (CHMs) have a long history of usage by Chinese medicinal practitioners in China and have been subjected to empirical investigations to determine its utility for treating T2DM. Several clinical trials [8-11] demonstrate the ability of CHMs to provide consistent glycemic control along with added benefits such as decreasing blood glucose levels, ameliorating insulin resistance and boosting pancreatic islets function, promoting weight loss, and having a low incidence of adverse events. Pharmacological studies demonstrated that CHMs may have the potential to partially restore islet beta cell function lost due to trauma [12-14], increase insulin secretion $[15,16]$, and strengthen peripheral glucose uptake $[17,18]$.

Given the significant medical and socioeconomic burden of T2DM, several controlled studies were performed to explore the safety and efficacy of CHMs for T2DM. A 2004 Cochrane Review [19] evaluated the efficacy of CHMs for T2DM and reported that certain compounds may be able to lower blood glucose levels in T2DM. However, these findings should be interpreted with caution due to the small number of trials with low methodological quality. High-quality trials are warranted to properly investigate the utility of CHMs in T2DM, and, indeed, several have been performed since then. This systematic review and meta-analysis aims to evaluate the safety and efficacy of CHMs for treating patients with T2DM. With this data, we are able to better conclude if CHMs is able to function as a complementary therapy for T2DM and to clarify whether CHMs in combination with antidiabetic agents or CHMs treated alone are able to confer a hypoglycemic effect in T2DM.

\section{Methods}

The Preferred Reporting Items for Systematic Reviews and Meta Analyses (PRISMA) guidelines were used to guide the execution and reporting of this review. Systematic review registration no. is PROSPERO (CRD42018093867). This work does not need ethics approval due to requirement of PRISMA (Supplementary File 1).

2.1. Database and Search Strategies. Relevant reports were extracted from the following databases: MEDLINE, EMBASE, PUBMED, the Cochrane Library, China National Knowledge Internet, Global Health, International Pharmaceutical Abstracts, Web of Science and the China biology medicine, Wanfang, and VIP databases. The literature search strategy (Supplementary File 2) was constructed based on the following facets: the study design (randomized clinical trial), the intervention (Chinese herbal medicine), and the condition (T2DM). The search terms utilized were (herbal medicine OR herbs OR Chinese herbal medicine OR Chinese medicinal herb OR Chinese patent medicine) and (type 2 diabetes mellitus OR type 2 diabetes OR 2 diabetes OR DM) and (randomized clinical trial OR randomized OR RCT). Journals published either in Chinese or English were included in the final results.

\subsection{Inclusion Criteria}

2.2.1. Types of Studies. All studies selected were designed to be clinical randomized controlled trials (RCTs).

2.2.2. Types of Participants. Participants were of any ethnic origin, gender, and age and the definition of T2DM used in the studies was required to be in accordance with either the World Health Organization criteria [20] or the American Diabetes Association criteria [6].

2.2.3. Types of Interventions. Participants in all trials either received $\mathrm{CHMs}$ treatment or a control medication. Patients in the CHMs treatment received CHMs + antidiabetic agents while those in the control group received either a placebo + antidiabetic agents or antidiabetic agents in isolation. Medication dosages of drugs across both intervention and control groups were required to be similar.

2.2.4. Types of Outcome Measures. The primary measures of outcomes of interest were HbAlc (glycosylated hemoglobin) after treatment. Secondary outcome measurements were 2hour postprandial blood glucose (2hPG), fasting blood glucose (FBG), and Body Mass Index (BMI). Medication safety data, if reported by the trial, was defined as the frequency of adverse events that occurred during the trial as a result of the medication.

2.3. Data Extraction and Management. Three independent authors (Jin De, Jiaxing Tian, and Qi Bao) screened the titles, abstracts, and contexts of each study for inclusion/exclusion with identical selection criterion. Incongruent opinions were first discussed, with a third party (Fengmei Lian) consulted for further resolution of any disagreements. The following data were extracted: trial characteristics (title, authors, year); baseline population characteristics (sample size, gender, age); interventions (treatment and control choice, dosage, and regimen); and outcomes (outcome measures, duration of patient follow-up, adverse events)

2.4. Statistical Analysis. The RevMan5.2 software provided by the Cochrane Collaboration was used to pool all reported outcomes [21]. Categorical outcomes were analyzed to determine relative risks (RRs) and accompanying 95\% Cis; continuous outcomes were analyzed to determine WMDs (weighted mean differences) and accompanying 95\% confidence intervals (CIs). Study heterogeneity was assessed with the I2 statistical method. If the I 2 value was $<50 \%$, the fixed-effects (FE) model was adopted; values of $<50 \%$ were subjected to the random-effect model. Corresponding authors were 


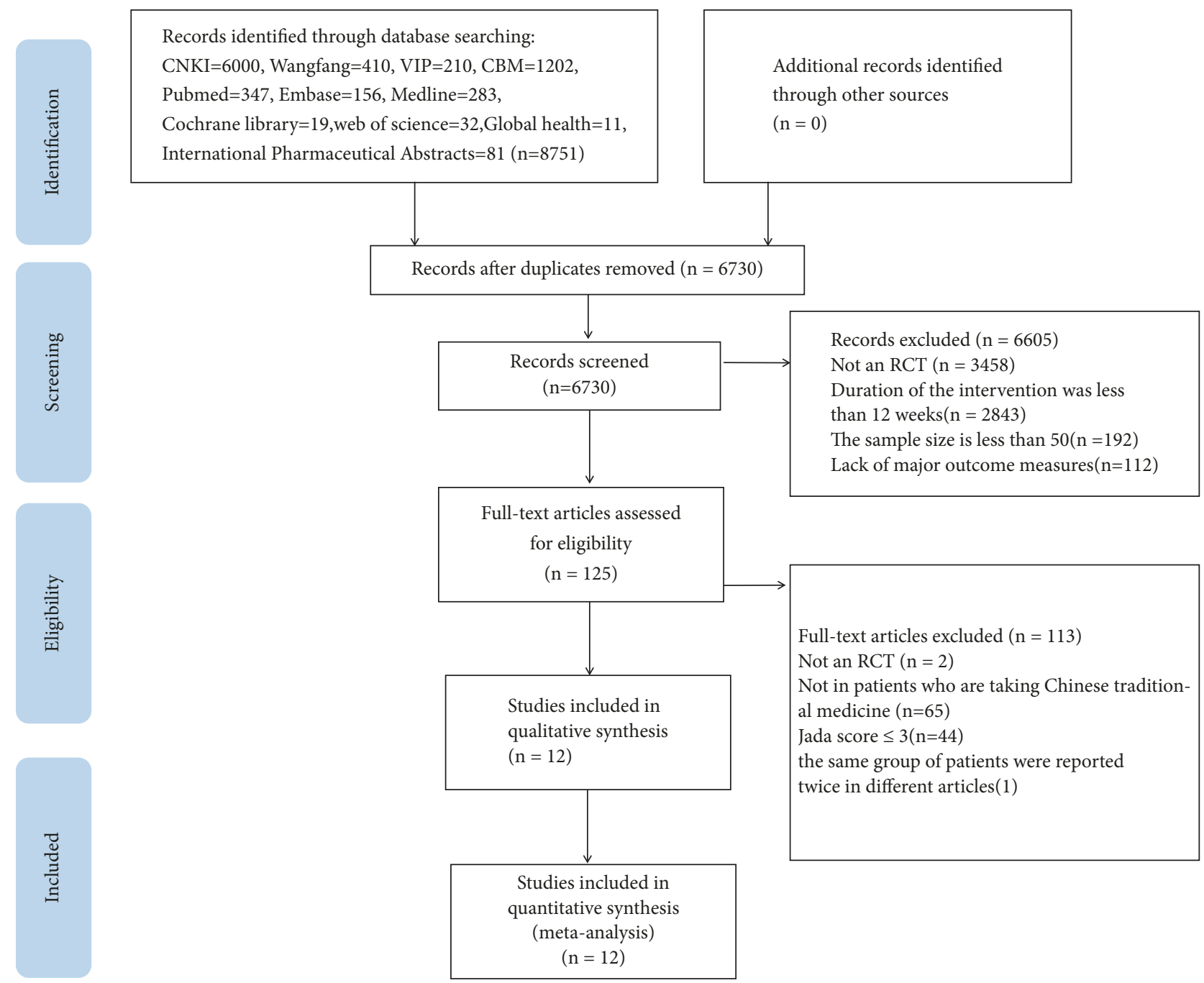

FIGURE 1: The screening process summarized in a flow diagram.

contacted directly for clarification should there be incomplete or missing primary outcome data (e.g., standard deviation (SD) and variance measures). When necessary, SD values were derived from SE or CI based on methods outlined in the Cochrane Handbook. Potential publication bias was assessed via a funnel plot.

2.5. Study Selection. A total of 6730 potentially relevant articles were identified. A total of 6605 articles were excluded on the basis of study design, a short duration of intervention of less than 3 months, a lack of major outcome measures or a lack of a comparison group. Out of these 6730 articles, we evaluated 125 full-text articles for inclusion. 115 articles were excluded as they were not designed as RCT, not utilizing CHMs or were duplicates. A final count of 10 RCTs comprising 2004 participants met our inclusion criteria $[8,10,11,22-28]$. Figure 1 depicts the screening process in the form of a flow diagram (Figure 1).
2.6. Characteristics of Included Studies. 2004 participants between the age of 18 and 62 years old were included in all eligible RCTs. Table 1 presents a summary of the characteristics of the studies, including author and publication year, sample size, participant age, course of disease, intervention and dosage, treatment duration, and outcome measures. All ten trials contrasted two groups of patients, one of which was designated as the "treatment group" (abbreviated to "T"in Table 1; these subjects were treated with CHMs + antidiabetic agents) and the other being a "control group"(abbreviated to "C"in Table 1; these subjects were treated with antidiabetic agents + placebo or antidiabetic agents only).

2.7. Methodological Quality Assessment and Evidence Quality. Two independent reviewers (Hai-yu Zhang and Qiyou Ding) reviewed the quality of each included trial based on the Cochrane Collaboration's tool to assess the risk of bias. Randomized controlled trials were assessed using the Cochran 







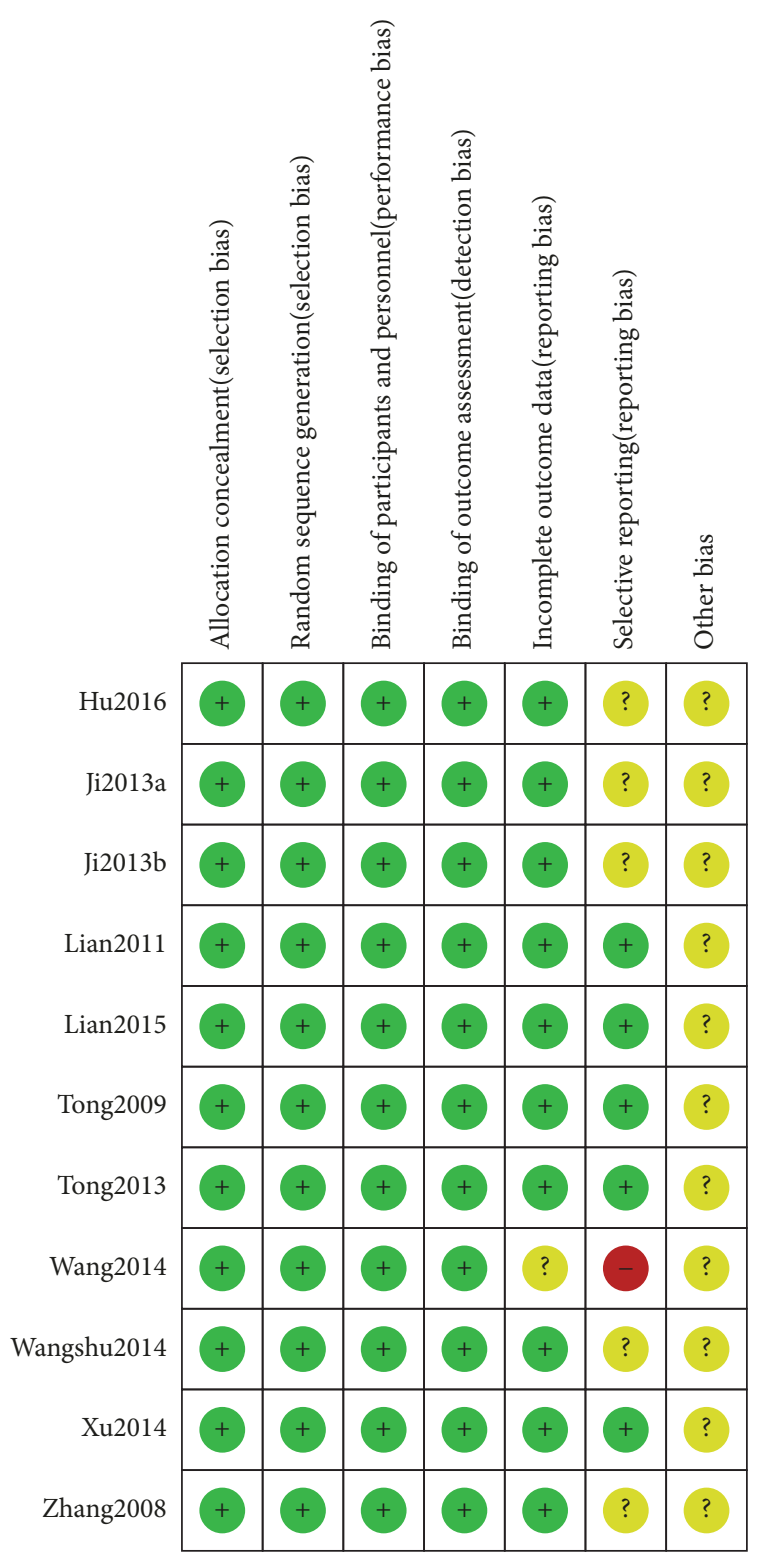

FIGURE 2: Quality assessment of the included trials-risk of bias graph.

Risk of Bias tool [29] which evaluates random sequence generation, allocation concealment, blinding, incomplete data regarding outcome, selective reporting, and other bias. All criteria on the list were ranked on a scale of low, unclear, or high bias risk. Any disputes between analyses were discussed until resolution was achieved. Figures 2 and 3 depict a detailed overview of how each study scored in each category of bias. Grading of Recommendations Assessment, Development and Evaluation (GRADE) [30] was used to assess the quality of the evidence for each outcome. According to GRADE, RCTs are considered higher-quality evidence, and observational studies are of lower quality. The risk of bias (in individual studies), inconsistency (heterogeneity in estimates of effect across studies), indirectness (related to the question or due to intransitivity), imprecision, and publication bias were addressed (Figure 8).

2.8. Description of the CHMs. Table 2 depicts a summary of all types of CHMs utilized across the included studies. 50 different types of CHMs were described. The top 16 most frequently utilized herbs that were used more than three times across the studies were goldthread root (Coptis chinensis), Rehmannia glutinosa (Rehmanniae Radix Praeparata), Panax ginseng (Panax Ginseng C.A.Mey.), Lobed Kudzuvine Root (Radix Puerariae), Milkvetch Root (Hedysarum Multijugum Maxim.), Trichosanthes kirilowii Maxim(Trichosanthis Radix), Bupleurum chinense (Radix Bupleuri), Rhubarb (Radix Rhei Et Rhizome), Lycium chinense Mill root (Lycii Cortex), Wolfiporia cocos (Poria Cocos(Schw.) Wolf.), Scutellaria baicalensis (Scutellaria baicalensis Georgi), nagaimo (Dioscorea opposita), Cornus officinalis Sieb. et Zucc (Cornus officinalis), Atractylodes Lancea (Thunb.) DC. (Rhizoma Atractylodis), Prunus mume (Dark Plum Fruit), and Rhizoma Anemarrhenae (Anemarrhena asphodeloides) (Table 3).

\subsection{Meta-Analysis}

2.9.1. Change in $\mathrm{HbAlc}$. Figure 4 depicts the change in $\mathrm{HbAlc}$ values. All 10 trials $(n=2004)$ reported treatment groups to have significantly changed HbAlc in contrast to the control groups. All HbAlc levels were relatively homogenous across trials, allowing for a fixed-effects model to be employed for statistical analysis. Change in HbAlc caused by CHMs was compared to the corresponding HbAlc values in the control group via subgroup analysis. The impact of CHMs on HbAlc levels were significantly different $(n=912 ; M D$, $0.48 ; 95 \% \mathrm{CI},-0.63$ to $-0.32 ; \mathrm{p}<0.00001 ; \mathrm{I} 2=39 \%$ ) compared to patients using placebos alone in 5 trials. The remaining five trials (indicated as "Add on") compared change in HbAlc conferred by use of either CHMs + antidiabetic drugs or placebo + antidiabetic drugs $(n=1092)$. These trials also demonstrated that a combination of CHMs + antidiabetic drugs was able to result in significantly reduced HbAlc levels (MD, $-0.22 ; 95 \% \mathrm{CI},-0.36$ to $-0.08, \mathrm{p}<0.01 ; \mathrm{I} 2=32 \%)$ in contrast to placebo.

2.9.2. FBG. FBG levels are contrasted in Figure 5. All 10 trials $(n=2004)$ included FBG levels as an outcome. Given that the results between trials were significantly heterogenous, a random-effects model was used to analyze trials. Subgroup analyses were performed to contrast FBG level differences between the treatment and control groups. In five trials, CHMs were found to significantly attenuate FBG levels in comparison to the control group $(n=912 ; M D,-0.69 ; 95 \% \mathrm{CI}$, -0.96 to $-0.43 ; \mathrm{p}<0.00001 ; \mathrm{I} 2=40 \%)$. Subsequent analysis of the remaining five trials found that a combination of $\mathrm{CHMs}$ + antidiabetic drugs yielded significant differences in FBG levels when compared to patients taking a combination of placebo + antidiabetic drugs ( $\mathrm{n}=1092 ; \mathrm{MD},-0.39 ; 95 \% \mathrm{CI}$, -0.78 to $-0.01 ; \mathrm{p}=0.04 ; \mathrm{I} 2=47 \%)$.

2.9.3. 2hPG. 2-hour PG levels are compared in Figure 6. $2 \mathrm{hPG}$ levels were evaluated in 8 trials. A random-effects 


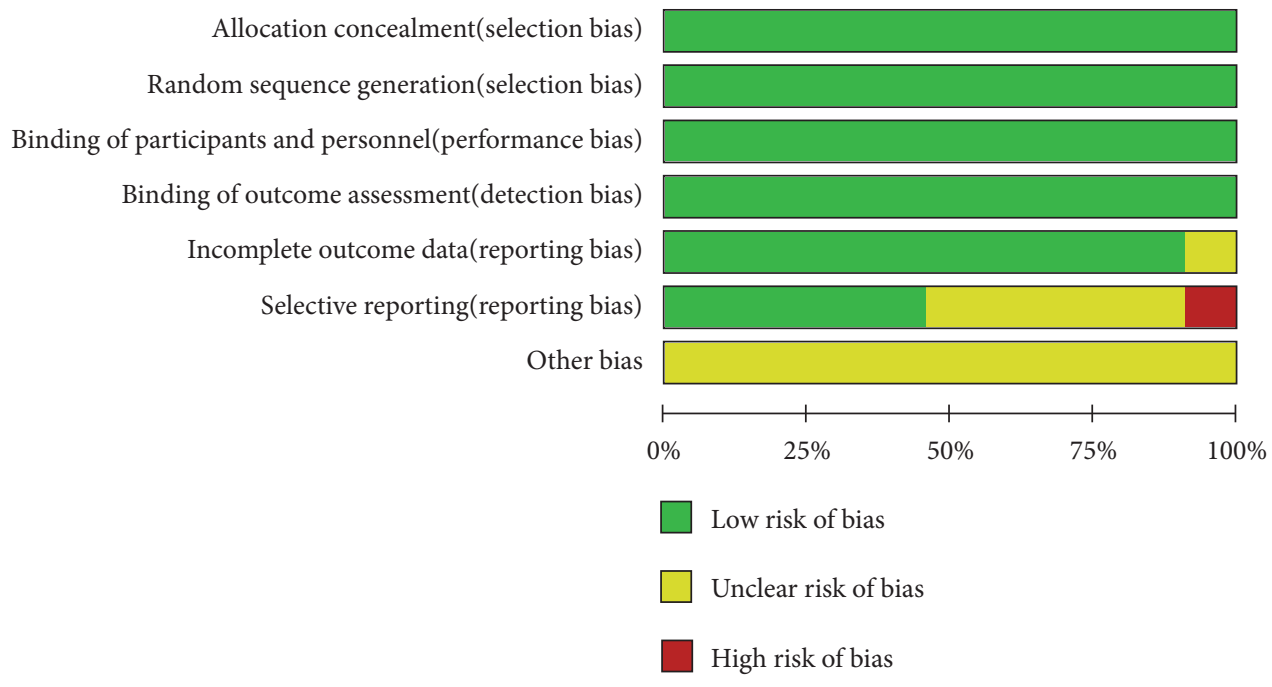

FIGURE 3: Quality assessment of the included trials-risk of bias summary.

TABLE 2: Analysis of the top 16 frequently used Chinese herb medicines in treatment of $\mathrm{T}_{2} \mathrm{DM}$.

\begin{tabular}{lccc}
\hline Herb name English (Latin) & Frequency & frequency \% & Cumulative percentiles \% \\
\hline goldthread root (Coptis chinensis) & 7 & 8.64 & 8.64 \\
Rehmannia glutinosa (Rehmanniae Radix Praeparata) & 5 & 6.17 & 14.81 \\
Panax ginseng (Panax Ginseng C. A. Mey.) & 5 & 6.17 & 20.98 \\
Lobed Kudzuvine Root (Radix Puerariae) & 3 & 3.70 & 24.68 \\
Milkvetch Root (Hedysarum Multijugum Maxim.) & 3 & 3.70 & 28.38 \\
Trichosanthes kirilowii Maxim (Trichosanthis Radix) & 3 & 3.70 & 32.08 \\
Bupleurum chinense (Radix Bupleuri) & 2 & 2.47 & 34.55 \\
Rhubarb (Radix Rhei Et Rhizome) & 2 & 2.47 & 37.02 \\
Lycium chinense Mill root (Lycii Cortex) & 2 & 2.47 & 39.49 \\
Wolfiporia cocos (Poria Cocos(Schw.) Wolf.) & 2 & 2.47 & 41.96 \\
Scutellaria baicalensis (Scutellaria baicalensis Georgi) & 2 & 2.47 & 44.43 \\
nagaimo (Dioscorea opposita) & 2 & 2.47 & 46.90 \\
Cornus officinalis Sieb. et Zucc (Cornus officinalis) & 2 & 2.47 & 49.37 \\
Atractylodes Lancea (Thunb.)DC.(Rhizoma Atractylodis) & 2 & 2.47 & 51.84 \\
Prunus mume (Dark Plum Fruit) & 2 & 2.47 & 54.31 \\
Rhizoma Anemarrhenae (Anemarrhena asphodeloides) & 2 & 2.47 & 56.78 \\
\hline
\end{tabular}

model was utilized for statistical analysis as there was significant result heterogeneity across trials. The impact of treatment and control groups of $2 \mathrm{hPG}$ levels were further assessed via subgroup analyses. $2 \mathrm{hPG}$ levels were compared between CHMs and placebo alone in eight trials, with results favoring treatment with CHMs ( $\mathrm{n}=912 ; \mathrm{MD},-1.07 ; 95 \%$ $\mathrm{CI},-1.69$ to $-0.46 ; \mathrm{p}=0.0007 ; \mathrm{I} 2=36 \%)$. $2 \mathrm{hPG}$ levels were also significantly different between groups using CHMs + antidiabetic drugs and groups using placebo + antidiabetic drugs $(\mathrm{n}=355 ; \mathrm{MD},-1.80 ; 95 \% \mathrm{CI},-2.72$ to $-0.89 ; \mathrm{p}=0.0001$; $\mathrm{I} 2=0 \%)$.

2.9.4. BMI. BMI data from six studies appear in Figure 7 and comparisons of BMI with CHMs and antidiabetic agents with controls showed heterogeneity between trials (I2=0\%) and a statistical difference between two groups for BMI variations ( $\mathrm{n}=1654, \mathrm{MD}=-0.04$; 95\% CI-0.70 to -0.19) (Figure 7).

2.10. Adverse Events. Adverse events were reported in four trials, with two of these reporting the absence of adverse events (Table 4). Statistical analysis of the two trials that reported adverse events found no significant differences between the rate of adverse events between the treatment and placebo groups $(n=1224$, RR:0.70; 95\% CI 0.37-1.29). Routine blood, renal, liver, and urine analysis as well as ECG findings demonstrated no significant difference between preand posttreatment values.

2.11. Assessment of Quality of Evidence. Table 5 showed overall evidence quality for each outcome (except adverse events) 
TABLE 3: Herbal medicines in the included studies.

\begin{tabular}{|c|c|c|c|c|}
\hline $\begin{array}{l}\text { First author } \\
\text { year }\end{array}$ & $\begin{array}{l}\text { Name of } \\
\text { Herbs }\end{array}$ & Formulation & Compositions (Latin) & Usage \\
\hline Xu 2014 & $\begin{array}{c}\text { Gegen } \\
\text { Qin-lian tang }\end{array}$ & decoction & $\begin{array}{l}\text { Radix Puerariae, Picrorhizae Rhizoma, Scutellariae } \\
\text { Radix, licorice }\end{array}$ & 200ml, Bid \\
\hline Tong 2009 & $\begin{array}{l}\text { Tang } \\
\text { Ming-ling } \\
\text { pills } \\
\end{array}$ & pills & $\begin{array}{c}\text { Radix Bupleuri, Scutellariae Radix, Radix Rhei Et } \\
\text { Rhizome, Aurantii Fructus Immaturus, Picrorhizae } \\
\text { Rhizoma, Arum Ternatum Thunb. }\end{array}$ & $6 \mathrm{~g}$, Tid \\
\hline $\begin{array}{l}\text { Wangshu } \\
2014\end{array}$ & $\begin{array}{c}\text { Tang Ke Soft } \\
\text { Capsules }\end{array}$ & capsules & Schisandrae Sphenantherae Fructus & 1 capsule, Bid \\
\hline Zhang 2008 & $\begin{array}{l}\text { Berberine } \\
\text { tablets }\end{array}$ & tablets & Berberin & $0.5 \mathrm{~g}, \mathrm{Bid}$ \\
\hline Tong 2013 & $\begin{array}{c}\text { Tang } \\
\text { Ming-ling } \\
\text { pills(TM81) } \\
\end{array}$ & pills & $\begin{array}{c}\text { Radix Bupleuri, Scutellariae Radix, Radix Rhei Et } \\
\text { Rhizome, Aurantii Fructus Immaturus, Picrorhizae } \\
\text { Rhizoma, Arum Ternatum Thunb. }\end{array}$ & $6 \mathrm{~g}$, Tid \\
\hline Lian 2011 & $\begin{array}{l}\text { Tian Qi Jiang } \\
\text { Tang } \\
\text { Capsules }\end{array}$ & Capsules & $\begin{array}{l}\text { Hedysarum Multijugum Maxim., Trichosanthis } \\
\text { Radix, Fructus Ligustri Lucidi, Dendrobium } \\
\text { officinale Kimura et Migo, Panax Ginseng C. A. } \\
\text { Mey., Lycii Cortex, Picrorhizae Rhizoma, Cornus } \\
\text { Officinalis Sieb. Et Zucc., Ecliptae Herba, Galla } \\
\text { Chinensis } \\
\end{array}$ & 5 Capsules, Tid \\
\hline Wang 2014 & $\begin{array}{l}\text { Six- } \\
\text { ingredient } \\
\text { rehmannia } \\
\text { pill } \\
\text { and Folium } \\
\text { ginkgo tablet }\end{array}$ & tablets & $\begin{array}{c}\text { Rehmanniae Radix Praeparata, Cornus Officinalis } \\
\text { Sieb. Et Zucc.,Cortex Moutan, Rhizoma Dioscoreae, } \\
\text { Poria Cocos(Schw.) Wolf., Alisma Orientale (Sam.) } \\
\text { Juz.,Ginkgo Folium }\end{array}$ & $\begin{array}{l}\text { Six-ingredient } \\
\text { rehmannia pill, } \\
8 \text { pills, Tid } \\
\text { Folium ginkgo } \\
\text { tablet, } 2 \text { tablets, } \\
\text { Tid } \\
\end{array}$ \\
\hline Lian 2015 & $\begin{array}{l}\text { Jin li da } \\
\text { granules }\end{array}$ & granules & $\begin{array}{l}\text { Panax Ginseng C. A. Mey., Polygonati Rhizoma, } \\
\text { Picrorhizae Rhizoma, Sophorae Flavescentis Radix, } \\
\text { Ophiopogon japonicus (Linn. f.) Ker-Gawl, } \\
\text { Rehmanniae Radix Praeparata, Fallopia multiflora } \\
\text { (Thunb.) Harald, Cornus Officinalis Sieb. Et Zucc., } \\
\text { Poria Cocos(Schw.) Wol, Eupatorium Fortunei } \\
\text { Turcz, Picrorhizae Rhizoma, Anemarrhenae } \\
\text { Rhizoma, Epimrdii HerbafbfhRadix Salviae, Radix } \\
\text { Puerariae, Litchi Semen, Lycii Cortex }\end{array}$ & 9g/bag, Tid \\
\hline Ji2013a,b & Xiao Ke pills & pills & $\begin{array}{c}\text { Radix Puerariae, Rehmanniae Radix Praeparata, } \\
\text { Hedysarum Multijugum Maxim., Trichosanthis } \\
\text { Radix, Maydis Stigma, Schisandrae Sphenantherae } \\
\text { Fructus, Rhizoma Dioscoreae }\end{array}$ & 5 pills \\
\hline Hu 2016 & $\begin{array}{l}\text { Jian Yu Tang } \\
\text { Kang granule }\end{array}$ & tablets & $\begin{array}{c}\text { Hedysarum Multijugum Maxim, Rehmanniae Radix } \\
\text { Praeparata, Atractylodes Lancea (Thunb.)Dc., } \\
\text { Figwort Root, Picrorhizae Rhizoma, Euonymi Alati } \\
\text { Ramulus }\end{array}$ & 9 tablets, Tid \\
\hline
\end{tabular}

using the GRADE method. Generally, evidence quality was high for CHMs and diabetes, and FBG, 2hPG, and FBG

2.12. Publication Bias. A funnel plot was used to express the publication bias. There were 10 trials included in the funnel plot, and no significant asymmetry was observed (Figure 8).

\section{Discussion}

3.1. Summary of the Evidence and Explanation of the Results. Development of antidiabetic medication has expanded tremendously in the recent decades. The most common and most frequently studied oral antidiabetic agents are metformin, sulfonylureas, and thiazolidinediones. The Chinese
Diabetes Society (CDS), the American Diabetes Association (ADA), and the European Diabetes Association for the Study of Diabetes (EASD) all unanimously recommend the use of such agents for the initial treatment of T2DM [31]. The role of CHMs in primary healthcare in China and other Asian countries has been increasingly prominent [32$35]$, especially in the management of T2DM. A previous meta-analysis in 2002 Cochrane included 66 randomized controlled (8803 participants) studying the use of CHMs in type 2 diabetes; some herbal medicines show hypoglycaemic effects in type 2 diabetes. However, these articles with low methodological quality, small sample size, and lack of major evaluation outcomes (HbAlc) may add some difficulty in precisely evaluating the efficacy and safety of CHMs. We 
TABLE 4: Incidence of adverse events.

\begin{tabular}{lccc}
\hline & \multicolumn{2}{c}{ Total events/total number } & $\begin{array}{c}\text { Risk ratio } \\
(95 \% \text { CI })\end{array}$ \\
\hline Gastrointestinal reactions & Treatment & Control & $0.67(0.25,1.76)$ \\
Rash & $6 / 1109$ & $9 / 895$ & $0.33(0.44,3.11)$ \\
Weakness & $1 / 1109$ & $3 / 895$ & $1.00(0.15,6.87)$ \\
Weight loss & $2 / 1109$ & $2 / 895$ & $0.33(0.01,8.02)$ \\
Frequently urination & $0 / 1109$ & $1 / 895$ & $3.00(0.12,72.20)$ \\
Tinnitus & $1 / 1109$ & $0 / 895$ & $0.20(0.01,4.08)$ \\
Genital swelling & $0 / 1109$ & $2 / 895$ & $0.33(0.11,8.02)$ \\
Elevated blood white blood cell & $0 / 1109$ & $1 / 895$ & $0.33(0.11,8.02)$ \\
Decreased hemoglobin & $0 / 1109$ & $1 / 895$ & $5.00(0.25,102.0)$ \\
Elevated urine white blood cell & $2 / 614$ & $0 / 610$ & $1.00(0.06,15.62)$ \\
Total events & $1 / 614$ & $1 / 610$ & 20 \\
Incidence of any adverse event & 13 & & Pooled rate ratio: $0.70(0.37,1.29)$ \\
\hline
\end{tabular}

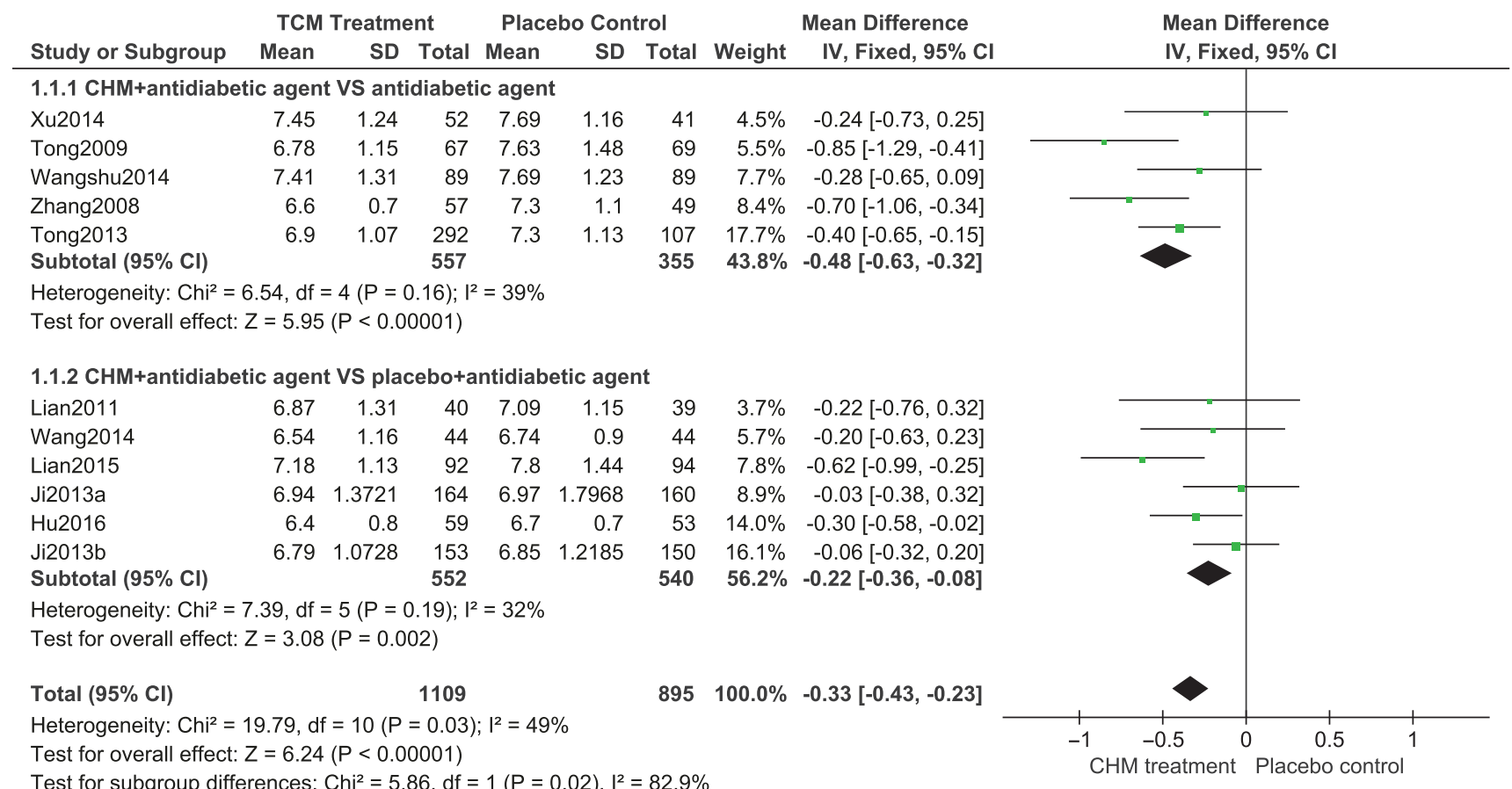

FIGURE 4: Forest plots of comparison of HAblc for CHM plus antidiabetic agents therapy versus antidiabetic agents alone.

performed a meta-analysis RCT (High Quality) to evaluate the efficacy and safety of CHMs plus antidiabetic agents in T2DM. Compared with the placebo + antidiabetic agents or antidiabetic agents alone, the CHMs plus antidiabetic agents typically reduced $\mathrm{HbAlc}, 2 \mathrm{hPG}$, and FPG levels in T2DM and resulted in a higher proportion of subjects that reduced weight.

Obesity or overweight is a risk factor for a wide range of chronic diseases, including coronary heart disease, diabetes, stroke, and cancer which associated with an increased risk of all causes of mortality. Weight control is necessary for patient with T2DM. Many antidiabetic drugs, such as thiazolidinediones, glinides, and sulphonylureas might lead to weight gain; it is vital for the patient with T2DM to select the appropriate drug. CHMs plus antidiabetic agents treatment resulted in an obvious reduction in BMI compared with the placebo + antidiabetic agents or antidiabetic agents monotherapy and might expand application range for antidiabetic drugs which might lead to weight gain.

In diabetes management, hypoglycemia is the most common adverse challenge. Interestingly, compared with the control group, the event of hypoglycemia was significantly lower in CHMs group than that in the placebo + antidiabetic agents or antidiabetic agents in subjects. The combination therapy was also associated with fewer adverse events (AEs). Our results showed the superiority of CHMs plus antidiabetic 


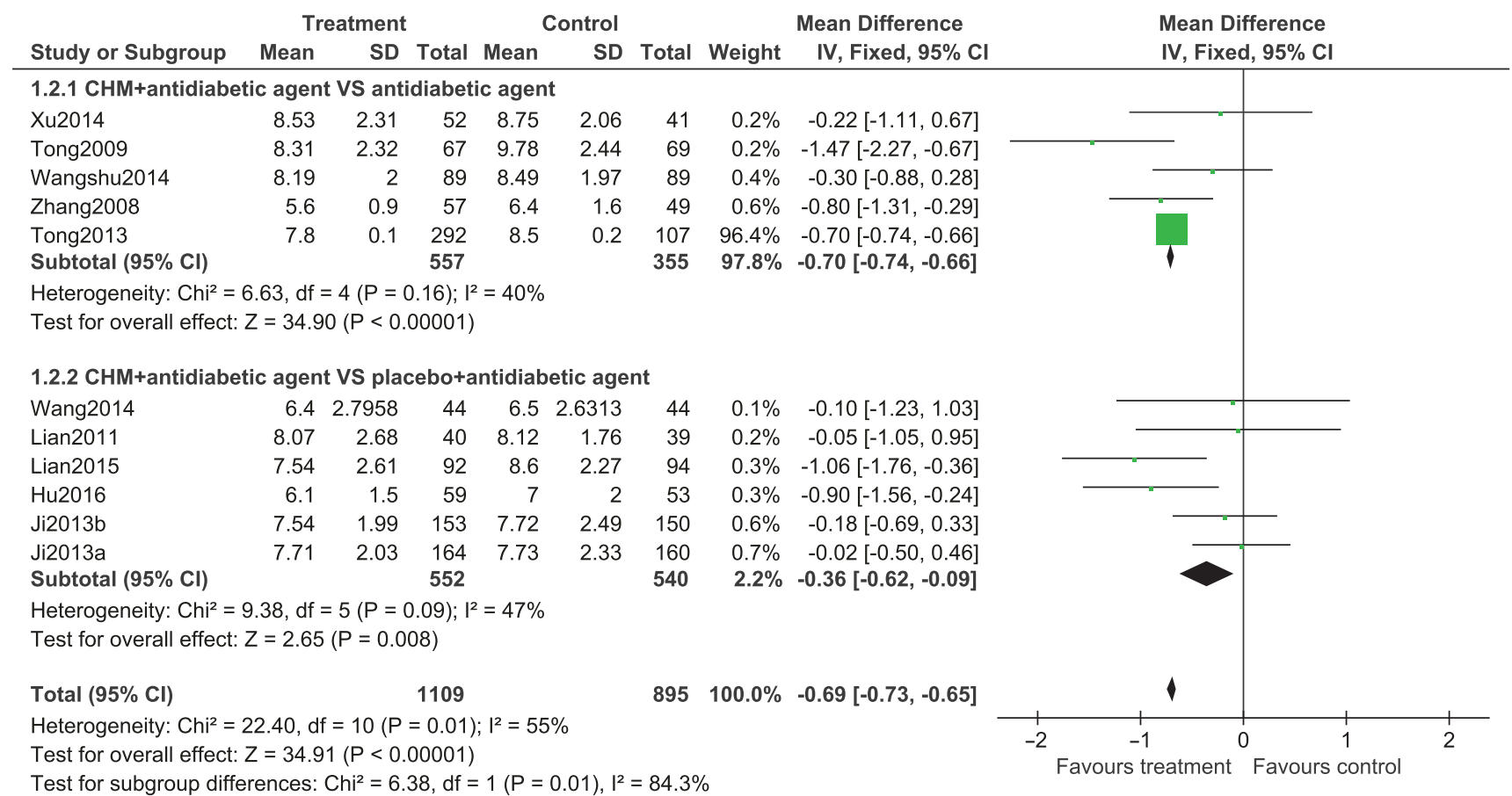

FIGURE 5: Forest plots of comparison of FBG for CHM plus antidiabetic agents therapy versus antidiabetic agents alone.

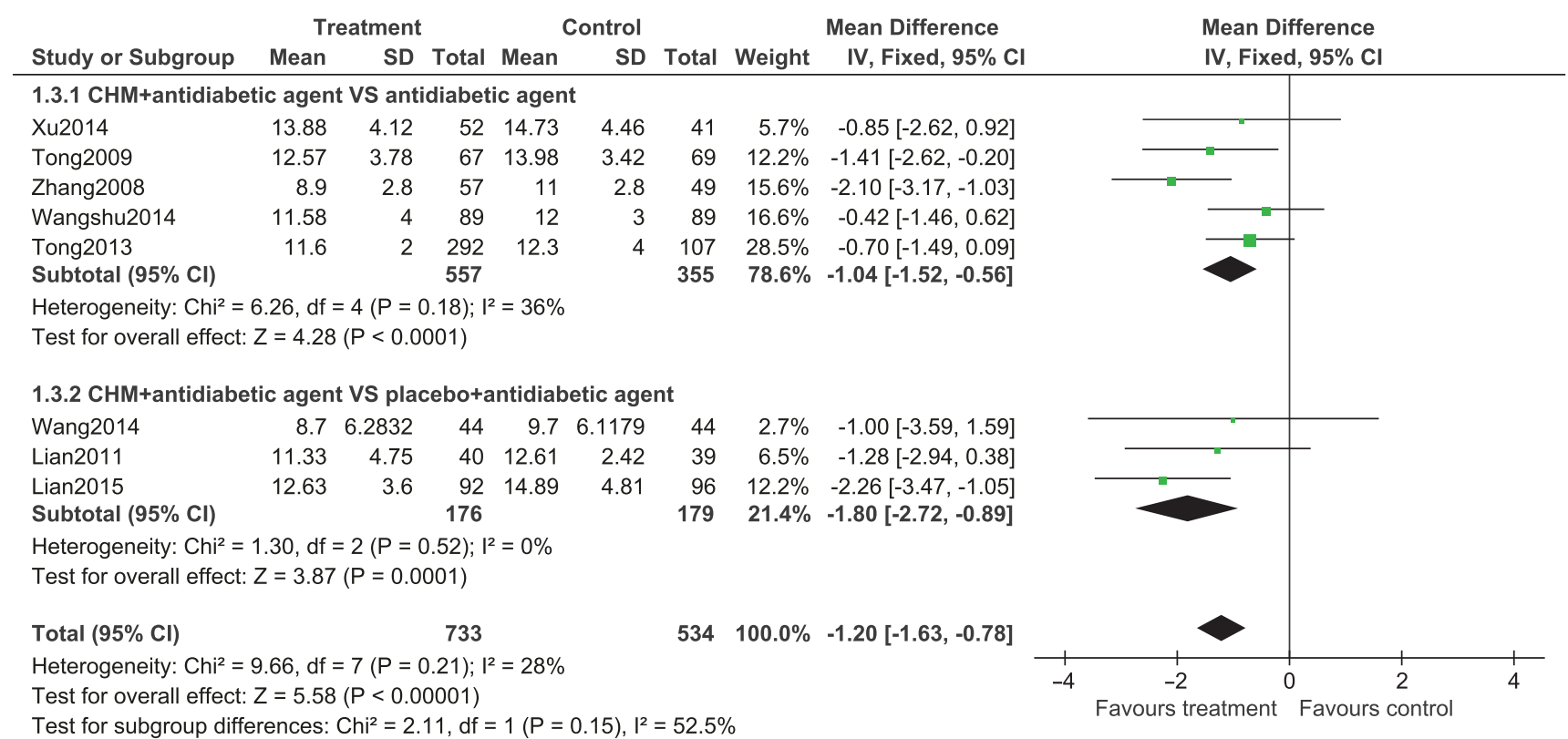

FIGURE 6: Forest plots of comparison of $2 \mathrm{hPG}$ for CHM plus antidiabetic agents therapy versus antidiabetic agents alone.

agents in patients with T2DM. CHMs are possibility beneficial when used as drug-combination therapy for patients with T2DM. However, the included antidiabetic drugs were only sulphonylureas, metformin, glinides, and thiazolidinediones. At present, there is lack of evidence associated with drug combination of other glucose-lowering drugs, such as GLP1, DPP-4, and SGLT2. More RCTs are needed to evaluate the efficacy and safety of CHMs plus newly developed hypoglycemic agents.

\section{Limitations}

The current systematic review and meta-analysis possesses a number of limitations. Firstly, trial registration with the WHO International Clinical Trials Registry Platform was not obtained for half of all included studies. In September 2007, the International Committee of Medical Journal Editors (ICMJE) began implementing a mandatory requirement that all clinical trials are to be registered [36]. Therefore, all trial 


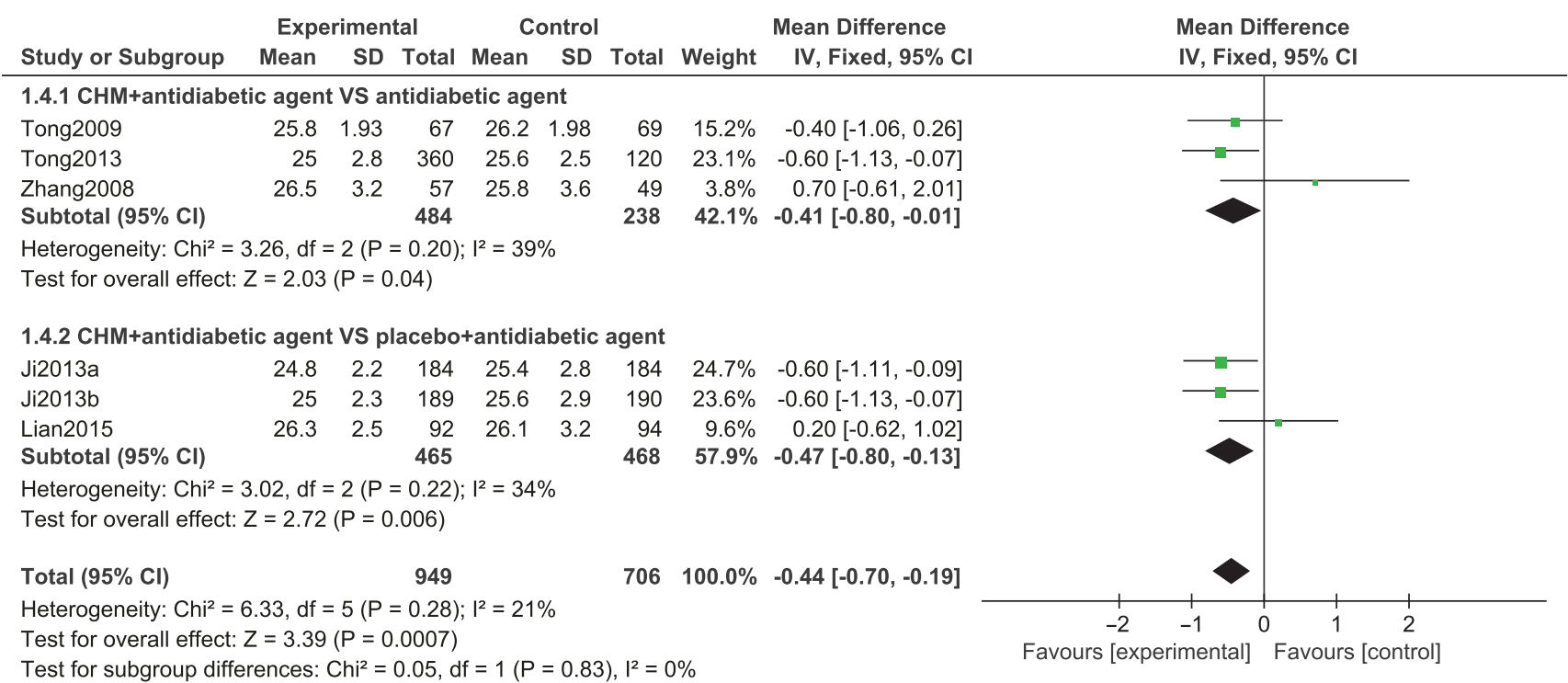

FIGURE 7: Forest plots of comparison of BMI for CHM plus antidiabetic agents therapy versus antidiabetic agents alone.

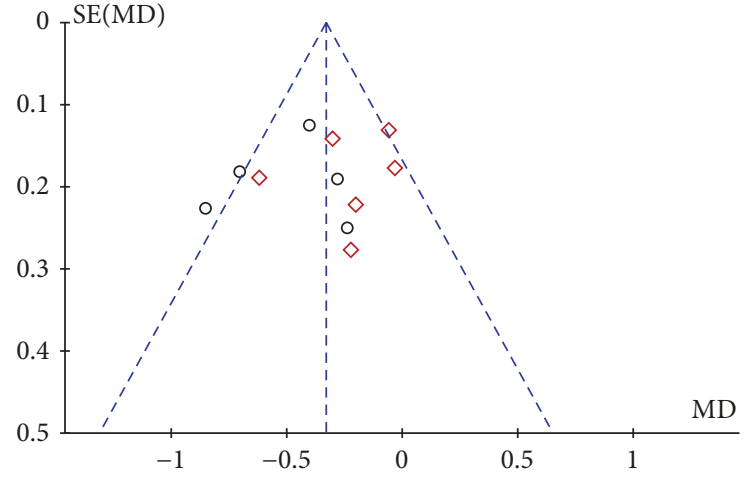

Subgroups

O CHM+antidiabetic agent VS antidiabetic agent

$\diamond \mathrm{CHM}+$ antidiabetic agent VS placebo+antidiabetic agent

FIGURE 8: Funnel plot of the trials that compared treatment group with control group.

protocols could not be confirmed to be free from biased reporting. Secondly, there was significant clinical heterogeneity across the included studies, with large variations in CHMs formulation, dosage, and treatment. Furthermore, there was insufficient detail available in the studies to adequately confirm manufacturing quality and therefore raised concerns regarding CHMs formulation consistency. Future studies would benefit from including descriptions of quality control, medication formulation, administration, and dosage [37].

Thirdly, several studies lacked formal estimation of sample sizes, leading to inadequate cohort sizes. This reduces the reliability and accuracy of obtained data and raises the risk of potentially overestimating the benefits of intervention $[38,39]$. Based on these reasons, results obtained from this meta-analysis should be interpreted with caution.
4.1. Implications for Practice. Based on the relative clinical heterogeneity of the included studies [I2 $=49 \%$, Test for overall effect: $\mathrm{Z}=6.24(\mathrm{P}<0.00001)]$ in reporting the effects of $\mathrm{CHMs}$ on HbA1c levels, along with the tolerability of CHMs across different patient cohorts, it could be concluded that CHMs may have the potential to serve as a safe, alternative, and complementary treatment for T2DM patients.

4.2. Implications for Research. $\mathrm{T} 2 \mathrm{DM}$ is a condition that is being increasingly managed with $\mathrm{CHMs}$, warranting larger clinical trials in this field. The most frequently used herbs such as goldthread root (Coptis chinensis), Rehmannia glutinosa (Rehmanniae Radix Praeparata), Panax ginseng (Panax Ginseng C.A.Mey.), Lobed Kudzuvine Root (Radix Puerariae), Milkvetch Root (Hedysarum Multijugum Maxim.), Trichosanthes kirilowii Maxim (Trichosanthis Radix), Bupleurum chinense (Radix Bupleuri), Rhubarb (Radix Rhei Et Rhizome), Lycium chinense Mill root (Lycii Cortex), Wolfiporia cocos (Poria Cocos (Schw.) Wolf.), Scutellaria baicalensis (Scutellaria baicalensis Georgi), nagaimo (Dioscorea opposita), Cornus officinalis Sieb. et Zucc (Cornus officinalis), Atractylodes Lancea (Thunb.) DC. (Rhizoma Atractylodis), Prunus mume (Dark Plum Fruit), Rhizoma Anemarrhenae (Anemarrhena asphodeloides) may contribute in the formation of a basic prescription for treating T2DM. Further trials should consider designing protocols in accordance with the CONSORT 2017 statement [40], a 25-item checklist assessing trial quality. Lastly, detailed information regarding CHMs preparations, quality of manufacturing, route of administration and dosage should be included for reference in future studies [37].

\section{Conclusion}

The current systematic review and meta-analysis has highlighted the benefits and safety of CHMs when used by patients with T2DM. CHMs treatment conferred clinically and statistically significant decreases in $\mathrm{HbAlc}, \mathrm{FPG}, 2 \mathrm{hPG}$, and 


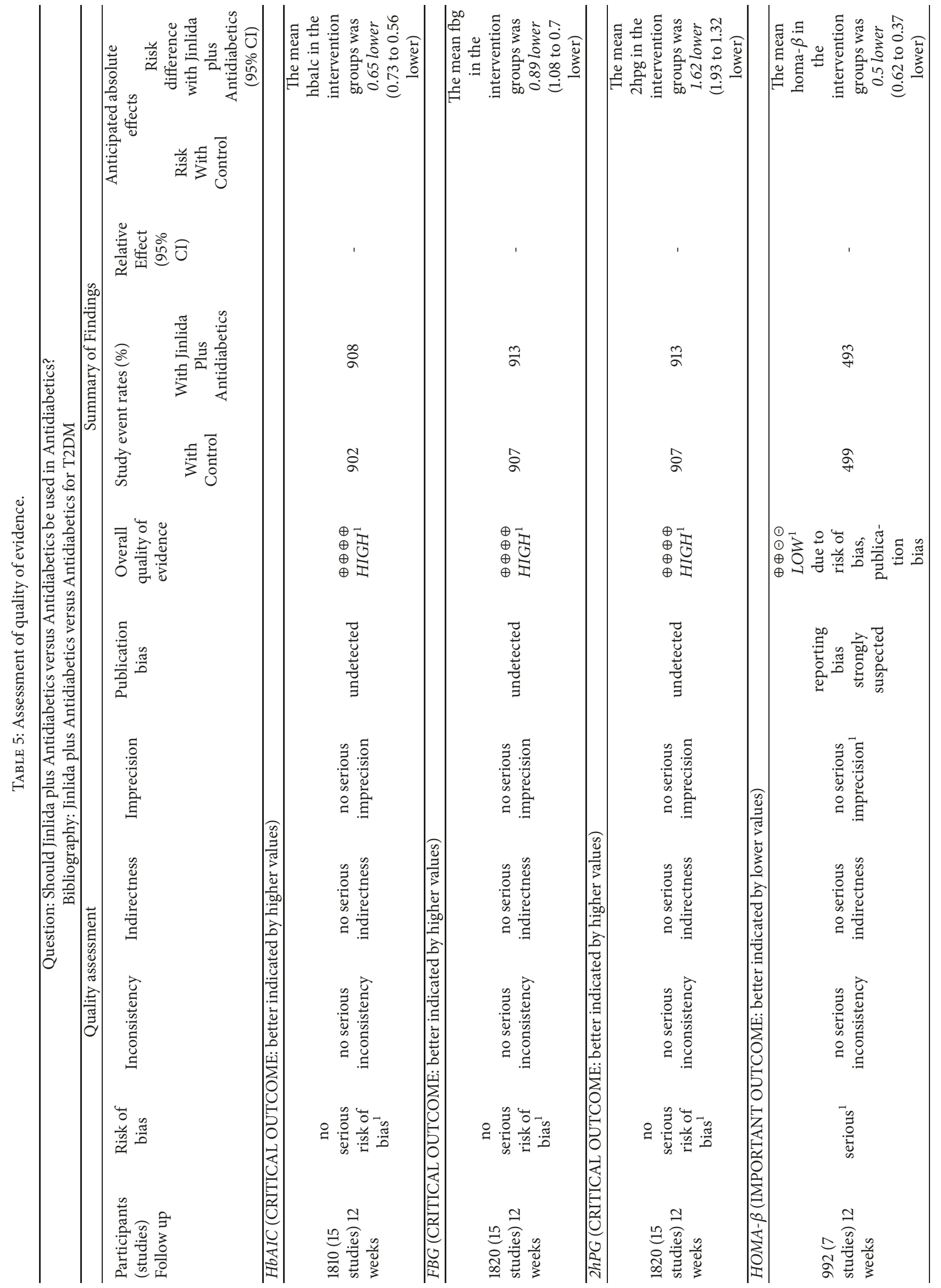




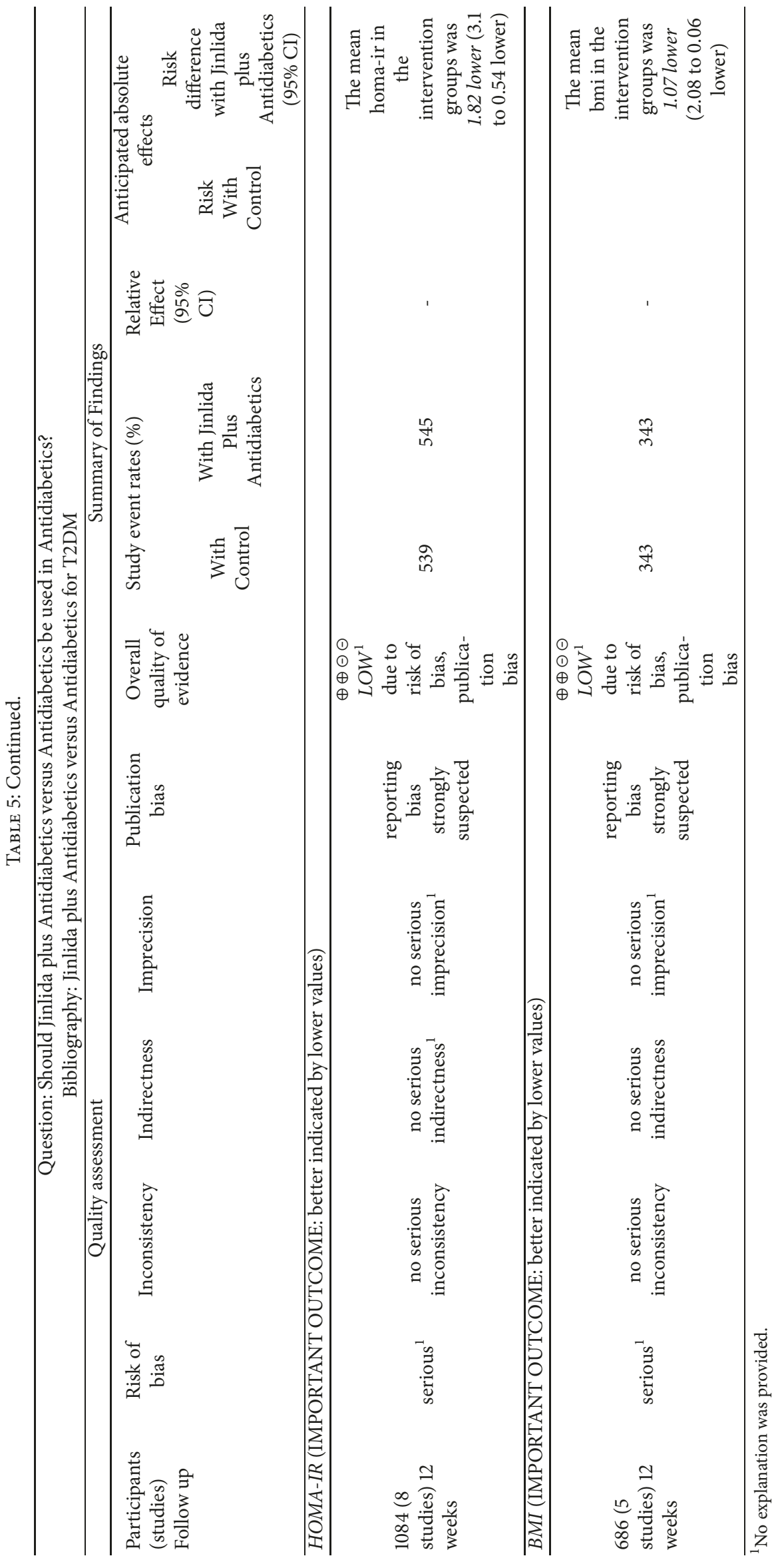


BMI levels in T2DM patients. Therefore, certain CHMs are possibly beneficial when used as drug-combination therapy for patients with T2DM. However, our findings should be interpreted with caution because of the limitations of the study. More rigorous RCTs are essential in allowing closer assessment of the potential benefits and safety profile of CHMs in the management of patients with T2DM.

\section{Conflicts of Interest}

All authors declare that there are no financial supports and relationships that may pose conflicts of interest.

\section{Authors' Contributions}

De Jin and Jiaxing Tian drafted the manuscript. Fengmei Lian and Tong Xiao-Lin revised the manuscript. Qi Bao and Haiyu Zhang searched all the literature and extracted data. Xiuping Chi and Qiyou Ding made statistical analysis of all the data. All of the authors participated in the design and read and approved the final manuscript, approving the published version and agreeing to be accountable for the accuracy and integrity. In this work, De Jin and Jiaxing Tian have equal contribution to this work as co-first authors and Fengmei Lian and Tong Xiao-Lin both are corresponding authors.

\section{Acknowledgments}

The authors would like to thank Xiuping Chi for critically reviewing the manuscript and making statistical analysis of all the data. This work was funded by the 2015 Traditional Chinese Medicine Scientific Research (no. 201507001-11) and supported by the Special Scientific Research Fund of Traditional Chinese Medicine Profession of China.

\section{Supplementary Materials}

Supplementary 1. Supplementary File 1: PRISMA 2009 Checklist.

Supplementary 2. Supplementary File 2: Literature retrieval strategy.

\section{References}

[1] L. Wang, P. Gao, M. Zhang et al., "Prevalence and ethnic pattern of diabetes and prediabetes in China in 2013," Journal of the American Medical Association, vol. 317, no. 24, pp. 2515-2523, 2017.

[2] W. T. Cade, "Diabetes-related microvascular and macrovascular diseases in the physical therapy setting," Physical Therapy in Sport, vol. 88, no. 11, pp. 1322-1335, 2008.

[3] R. W. Grant and M. S. Kirkman, "Trends in the evidence level for the american diabetes association's 'standards of medical care in diabetes' from 2005 to 2014," Diabetes Care, vol. 38, no. 1, pp. 68, 2015.

[4] UK Prospective Diabetes Study (UKPDS) Group, "Intensive blood-glucose control with sulphonylureas or insulin compared with conventional treatment and risk of complications in patients with type 2 diabetes (UKPDS 33)," The Lancet, vol. 352, no. 9131, pp. 837-853, 1998.
[5] S. E. Inzucchi, R. M. Bergenstal, J. B. Buse et al., "Management of hyperglycemia in type 2 diabetes, 2015: a patient-centered approach: update to a position statement of the american diabetes association and the european association for the study of diabetes," Diabetes Care, vol. 38, no. 1, pp. 140-149, 2015.

[6] P. H. Marathe, H. X. Gao, and K. L. Close, "American diabetes association standards of medical care in diabetes 2017," Journal of Diabetes, vol. 9, no. 4, pp. 320-324, 2017.

[7] M. N. Cook, C. J. Girman, P. P. Stein, and C. M. Alexander, "Initial monotherapy with either metformin or sulphonylureas often fails to achieve or maintain current glycaemic goals in patients with Type 2 diabetes in UK primary care," Diabetic Medicine, vol. 24, no. 4, pp. 350-358, 2010.

[8] F. Lian, J. Tian, X. Chen et al., "The efficacy and safety of Chinese herbal medicine Jinlida as add-on medication in type 2 diabetes patients ineffectively managed by metformin monotherapy: a double-blind, randomized, placebo-controlled, multicenter trial," PLoS ONE, vol. 10, no. 6, Article ID e0130550, 2015.

[9] X. L. Tong, S. T. Wu, F. M. Lian et al., "The safety and effectiveness of TM81, a Chinese herbal medicine, in the treatment of type 2 diabetes: a randomized double-blind placebo-controlled trial," Diabetes, Obesity and Metabolism, vol. 15, no. 5, pp. 448454, 2013.

[10] L. Ji, X. Tong, H. Wang et al., "Efficacy and safety of traditional Chinese medicine for diabetes: a double-blind, randomised, controlled trial," PLoS ONE, vol. 8, no. 2, Article ID e56703, 2013.

[11] Y. Zhang, X. Li, W. D. Liu et al., "Treatment of type 2 diabetes and dyslipidemia with the natural plant alkaloid berberine," Journal of Clinical Endocrinology \& Metabolism, vol. 93, no. 7, p. 2559, 2008.

[12] C. R. Cederroth, M. Vinciguerra, A. Gjinovci et al., "Dietary phytoestrogens activate AMP-Activated protein kinase with improvement in lipid and glucose metabolism," Diabetes, vol. 57, no. 5, pp. 1176-1185, 2008.

[13] S. P. Li, G. H. Zhang, Q. Zeng et al., "Hypoglycemic activity of polysaccharide, with antioxidation, isolated from cultured Cordyceps mycelia," Phytomedicine, vol. 13, no. 6, pp. 428-433, 2006.

[14] C.-H. Jung, H.-M. Seog, I.-W. Choi, H.-D. Choi, and H.-Y. Cho, "Effects of wild ginseng (Panax ginseng C.A. Meyer) leaves on lipid peroxidation levels and antioxidant enzyme activities in streptozotocin diabetic rats," Journal of Ethnopharmacology, vol. 98, no. 3, pp. 245-250, 2005.

[15] M.-P. Lu, R. Wang, X. Song, X. Wang, L. Wu, and Q. H. Meng, "Modulation of methylglyoxal and glutathione by soybean isoflavones in mild streptozotocin-induced diabetic rats," Nutrition, Metabolism \& Cardiovascular Diseases, vol. 18, no. 9, pp. 618-623, 2008.

[16] H. S. Zhen, J. Liang, and F. Zhou, "Studies on anti-hyperglycemic effect of ethanolic extracts from gymnema sylvestre(Retz.)schult," Chinese Journal of Experimental Traditional Medical Formulae, 2007.

[17] H. Cao, M. M. Polansky, and R. A. Anderson, "Cinnamon extract and polyphenols affect the expression of tristetraprolin, insulin receptor, and glucose transporter 4 in mouse 3T3-L1 adipocytes," Archives of Biochemistry and Biophysics, vol. 459, no. 2, pp. 214-222, 2007.

[18] B. Qin, M. Nagasaki, M. Ren, G. Bajotto, Y. Oshida, and Y. Sato, "Cinnamon extract (traditional herb) potentiates in vivo insulin-regulated glucose utilization via enhancing insulin signaling in rats," Diabetes Research and Clinical Practice, vol. 62, no. 3, pp. 139-148, 2003. 
[19] J. P. Liu, M. Zhang, W. Y. Wang, and S. Grimsgaard, "Chinese herbal medicines for type 2 diabetes mellitus," Cochrane Database of Systematic Reviews, no. 3, Article ID CD003642, 2004.

[20] F. De Vegt, J. M. Dekker, C. D. A. Stehouwer, G. Nijpels, L. M. Bouter, and R. J. Heine, "The 1997 american diabetes association criteria versus the 1985 world health organization criteria for the diagnosis of abnormal glucose tolerance: poor agreement in the hoorn study," Diabetes Care, vol. 21, no. 10, pp. 1686-1690, 1998.

[21] F. Godlee, "The cochrane collaboration: deserves the support of doctors and governments," British Medical Journal, vol. 309, no. 6960, pp. 969-970, 1994.

[22] T. xiao-lin, F. Ni-Qing Lian et al., "Multi-center randomized controlled and double blind trial of Tang-min-ling pill $s$ in the treatmen tof type 2 diabetes mellitus," The Chinese Journal of Clinical Pharmacology, vol. 25, no. 02, pp. 104-108, 2009.

[23] F. Lian, L. Yao, X. Sun, D. Xiang, X. Xiao, and X. Tong, “The combined Tian Qi Jiangtang capsule and metformin treatment in patients with type 2 diabetes mellitus: a randomized, parallelgroup, muiticenter, double-blind and prospective study," Chinese Journal of Diabetes, vol. 19, no. 08, pp. 600-602, 2011.

[24] X. L. Tong, S. T. Wu, F. M. Lian et al., "The safety and effectiveness of TM81, a Chinese herbal medicine, in the treatment of type 2 diabetes: a randomized double-blind placebo-controlled trial," Diabetes Obesity Metabolism, vol. 15, no. 5, pp. 448-454, 2013.

[25] S. Wang, Z. Chang, and X. Jiang, "A randomized, doubleblind, multicenter clinical trial for tangke soft capsules in the treatment of type 2 diabetes," Chinese Traditional Patent Medicine, vol. 36, no. 10, pp. 2045-2048, 2014.

[26] Y. Wangyan-ping, A. Yinha, W. X. Wang et al., "Effect of Liu wei Di huang combined with Ginkgo Leaf on fractalkine in type 2 diabetic patients," Journal of Southeast University (Medical Science Edition), vol. 33, no. 02, pp. 121-125, 2014.

[27] J. Xu, F. Lian, L. Zhao et al., "Structural modulation of gut microbiota during alleviation of type 2 diabetes with a Chinese herbal formula," The ISME Journal, vol. 9, no. 3, pp. 552-562, 2015.

[28] Y. Hu, X. Zhou, P. Liu, B. Wang, D.-M. Duan, and D.-H. Guo, "A comparison study of metformin only therapy and metformin combined with Chinese medicine jianyutangkang therapy in patients with type 2 diabetes: A randomized placebo-controlled double-blind study," Complementary Therapies in Medicine, vol. 24, pp. 13-18, 2016.

[29] M. Viswanathan, M. T. Ansari, N. D. Berkman et al., "Assessing the risk of bias of individual studies in systematic reviews of health care interventions - methods guide for effectiveness and comparative effectiveness reviews," Agency for Healthcare Research and Quality, 2012.

[30] C. Mendoza, P. Kraemer, P. Herrera, P. Burdiles, D. Sepulveda, and E. Nunez, "Clinical guidelines using the grade system (grading of recommendations assessment development and evaluation)," Revista medica de Chile, vol. 145, no. 11, pp. 14631470, 2017.

[31] S. E. Inzucchi, R. M. Bergenstal, J. B. Buse et al., "Management of hyperglycaemia in type 2 diabetes: a patient-centered approach. Position statement of the American Diabetes Association (ADA) and the European Association for the study of diabetes (EASD)," Diabetologia, vol. 55, no. 6, pp. 1577-1597, 2012.

[32] A. F. Ceylan-Isik, R. M. Fliethman, L. E. Wold, and J. Ren, "Herbal and traditional Chinese medicine for the treatment of cardiovascular complications in diabetes mellitus," Current Diabetes Reviews, vol. 4, no. 4, pp. 320-328, 2008.

[33] X. Hu, J. Sato, G. Bajotto et al., "Goshajinkigan (Chinese herbal medicine niu-che-sen-qi-wan) improves insulin resistance in diabetic rats via the nitric oxide pathway." Nagoya Journal of Medical Science, vol. 72, no. 1-2, pp. 35-42, 2010.

[34] H. J. Kim, K. H. Chun, D. J. Kim, S. J. Han, Y. S. Kim, and J. T. Woo, "Utilization patterns and cost of complementary and alternative medicine compared to conventional medicine in patients with type 2 diabetes mellitus," Diabetes Research Clinical Practice, vol. 93, no. 1, pp. 115-122, 2011.

[35] A. Alqahtani, K. Hamid, A. Kam et al., "The pentacyclic triterpenoids in herbal medicines and their pharmacological activities in diabetes and diabetic complications," Current Medicinal Chemistry, vol. 20, no. 7, pp. 908-931, 2013.

[36] K. B. Thomas and C. Tesch, Clinical TrIal Disclosure: The Ongoing Debate on Public Registers for Clinical Trials, 2007.

[37] J. J. Gagnier, H. Boon, P. Rochon, D. Moher, J. Barnes, and C. Bombardier, "Reporting randomized, controlled trials of herbal interventions: an elaborated CONSORT statement," Annals of Internal Medicine, vol. 144, no. 5, pp. 364-367, 2006.

[38] L. L. Kjaergard, J. Villumsen, and C. Gluud, "Reported methodologic quality and discrepancies between large and small randomized trials in meta-analyses," Annals of Internal Medicine, vol. 149, no. 3, pp. 982-989, 2001.

[39] Y. Wang, C.-L. Xie, D.-L. Fu et al., "Clinical efficacy and safety of Chinese herbal medicine for Wilson's disease: A systematic review of 9 randomized controlled trials," Complementary Therapies in Medicine, vol. 20, no. 3, pp. 143-154, 2012.

[40] I. Boutron, D. G. Altman, D. Moher et al., "CONSORT statement for randomized trials of nonpharmacologic treatments: a 2017 update and a CONSORT extension for nonpharmacologic trial abstracts," Annals of Internal Medicine, vol. 167, no. 1, pp. 40-47, 2017. 


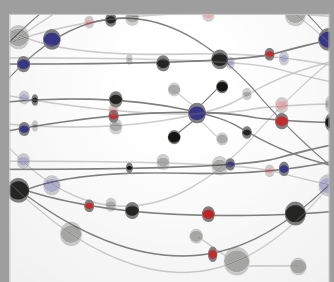

The Scientific World Journal
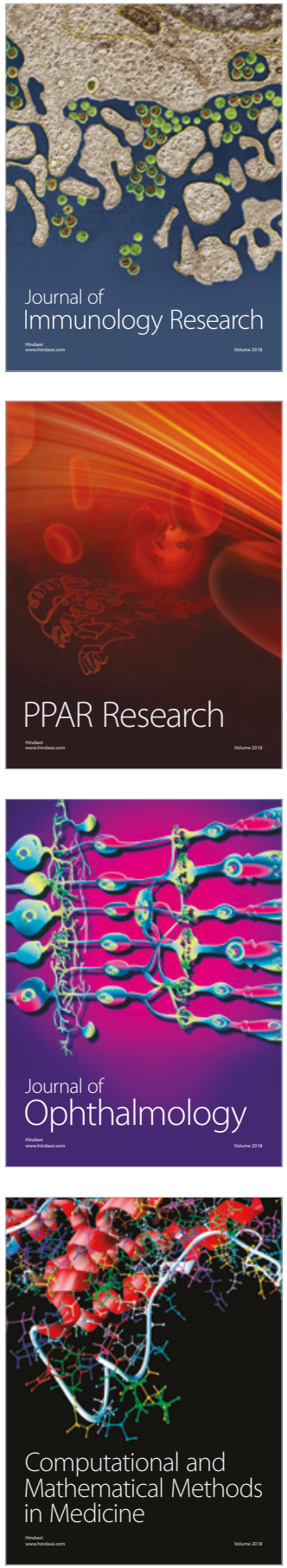

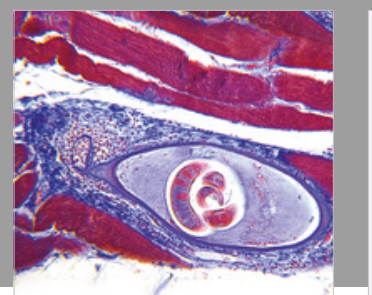

Gastroenterology Research and Practice

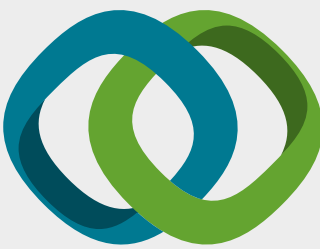

\section{Hindawi}

Submit your manuscripts at

www.hindawi.com
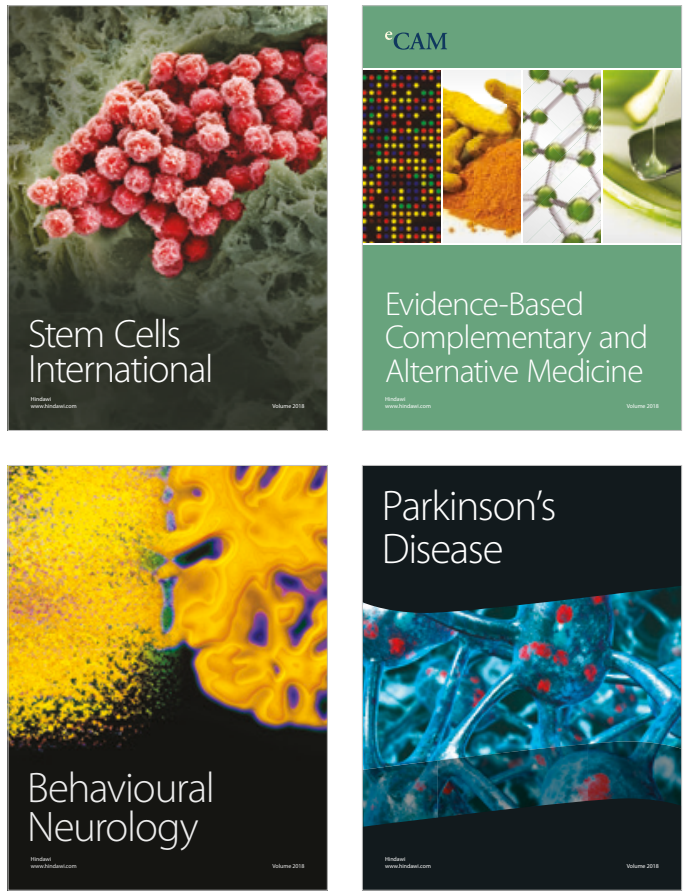

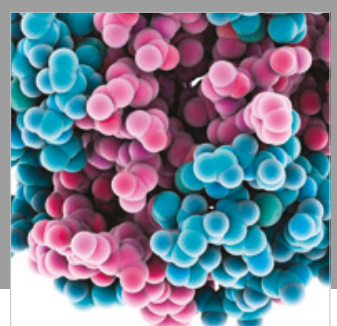

ournal of

Diabetes Research

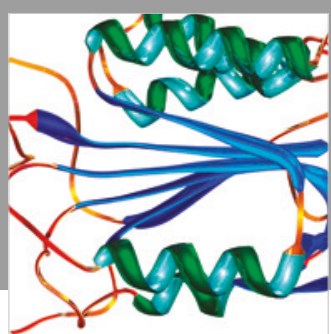

Disease Markers
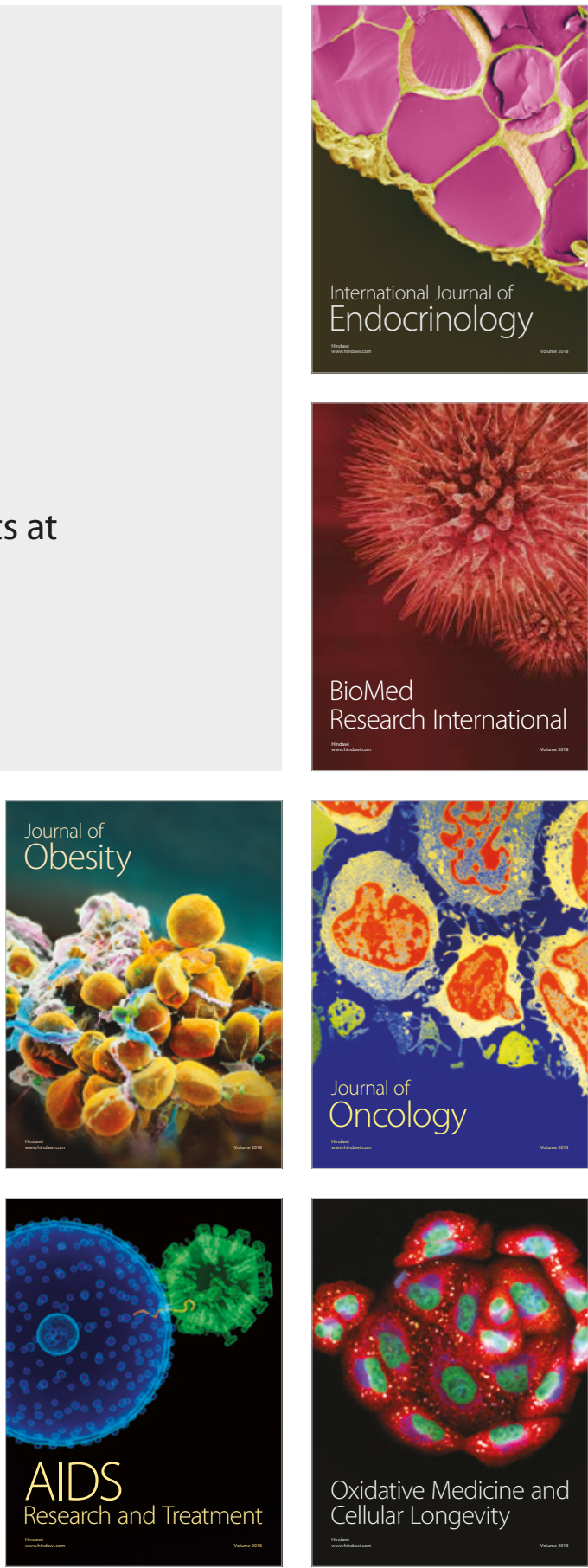